\title{
The Spontaneous-Rate Histogram of the Auditory Nerve Can Be Explained by Only Two or Three Spontaneous Rates and Long-Range Dependence
}

\author{
B. Scott Jackson and Laurel H. Carney \\ Institute for Sensory Research and Department of Bioengineering and Neuroscience, Syracuse University, \\ Syracuse, NY 13244, USA
}

Received: 2 August 2004; Accepted: 28 December 2004; Online publication: 10 June 2005

\begin{abstract}
Estimates of the spontaneous discharge rate (SR) of auditory-nerve (AN) fibers are often based on measurements of the average rate over a long (e.g., $30 \mathrm{~s}$ ) interval. These measurements are important because SR is apparently correlated with other AN properties, such as threshold to acoustic stimuli, shape of ratelevel function, recovery from prior stimulation, and certain anatomical characteristics. Furthermore, histograms of SR estimates from large numbers of fibers suggest that they can be divided into two (i.e., low and high) or three (i.e., low, medium, and high) SR classes. Yet, even "simple" statistical estimates, such as average rate, can behave surprisingly poorly for processes with long-range dependence (LRD), which has been found in the spontaneous activity of AN fibers. In particular, LRD greatly increases the variability of estimates of mean discharge rate. We investigated the implications of this effect of LRD for our understanding of the SRs of AN fibers. The fractional-Gaussian-noise-driven Poisson process (fGnDP) was originally developed to model the LRD action-potential trains of AN fibers. Using rate estimates computed from this model, we were able to reproduce the shape of published histograms of SR using only three fixed SR values. Moreover, by using a Poisson-equivalent integrate-and-fire (IF) model in place of the inhomogeneous Poisson process in the fGnDP model, we were able to reproduce SR histograms using only two fixed SR values. These results
\end{abstract}

Correspondence to: B. Scott Jackson - Cornell University • Seeley G. Mudd Hall, Ithaca, NY 14853, USA. Telephone: (607) 254-4384; fax: (607) 254-4308; email: bsj22@cornell.edu suggest that AN fibers may have only two or three possible values for their long-term average spontaneous discharge rates. In other words, all "high-SR" neurons may actually have the same underlying SR. Furthermore, both "low-SR" and "medium-SR" neurons may have a single "true" SR value, or these two classes may have two different "true" SR values. Furthermore, the Poisson-equivalent IF model may prove useful in other applications involving the modeling of trains of action potentials.

Keywords: long-range dependence, auditory nerve, spontaneous rate, average firing rate

\section{INTRODUCTION}

In this study we ask the question: How well does a histogram of estimates of spontaneous discharge rates (SRs) (e.g., Fig. 1) represent the distribution of the "true" SRs of auditory-nerve (AN) fibers? This histogram does not directly represent the distribution of the actual SRs but instead represents the distribution of estimated SRs. By "actual (or true)" SR, we denote the mean discharge rate that one would, theoretically, calculate from an infinitely long recording. This is the "population mean" discharge rate that is solely dependent on the properties of the neuron itself and is not the "sample mean" discharge rate that depends on when and how it is measured. The estimates of SR illustrated in Figure 1 are based on average discharge rates measured over 30-s intervals (Liberman 1978). If the variance of these average discharge rates is relatively small, then the histogram provides a good representation of the 


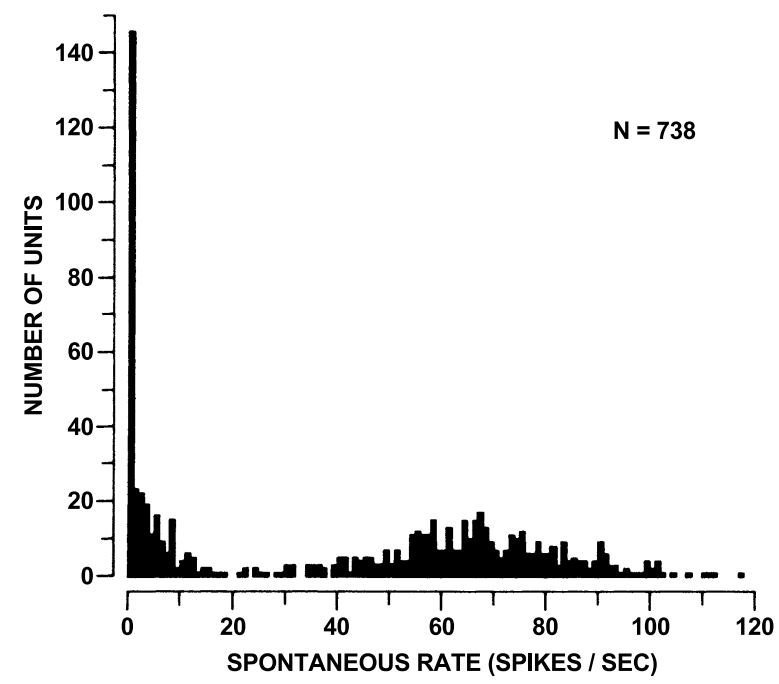

FIG. 1. Histogram of spontaneous rate estimates from 30-s recordings from 738 fibers in the auditory nerves of cats. The width of the bins is 1 spike/s. Reproduced with permission from Liberman (1978).

distribution of actual SRs. However, if the variance of the average discharge rates is large, then the histogram of the SR estimates may differ significantly from the actual distribution.

The question of how well the estimated SR histogram represents the actual distribution is not trivial because of the presence of long-range temporal dependence (LRD) in the trains of action potentials in AN fibers (Teich 1989; Teich and Lowen 1994; Lowen and Teich 1996a). The autocorrelations of an LRD process decay very slowly with increasing temporal lag. For a short-range-dependent process the infinite sum of these autocorrelations converges to a finite value. However, this infinite sum diverges for an LRD process. Essentially, this means that, for an LRD process, an infinite amount of the influence of the past on the present will necessarily be neglected when only a finite history of the process is known, regardless of the length of this finite history (see Beran 1994; Jackson 2003, 2004). It also means that statistical estimates from nearby time intervals will tend to be similar, because the influence of the history of the process will be very similar for these two intervals. Hence, errors in statistical estimates from one time interval will largely be reproduced in statistical estimates from other nearby intervals. Furthermore, for an LRD process, this effect decays very slowly as the distance between the two intervals is increased.

In previous studies (Jackson 2003), we found that LRD dramatically increases the variability of estimates of mean action-potential count. This study explores the implications of that finding for the interpretation of AN SR histograms. The broadness of these histograms suggests that AN fibers may have a broad range of SRs. However, the broadness of these histograms could also be due, at least in part, to variability in the action-potential counts used to estimate the SRs.

\section{METHODS}

The results presented below are based on simulations of spontaneous AN discharges. This section describes two procedures that were used to simulate discharge trains with appropriate LRD. The choices of model parameter values and the strategy for comparing simulation results to published SR histograms are also described here.

An LRD process has autocorrelations that decay so slowly that their infinite-time integral (or sum) diverges to infinity (see Beran 1994; Jackson 2003, 2004 for more details about the definition of LRD). In order to investigate the behavior of empirical average discharge rate calculations in LRD discharge trains, such as those in the AN, we would need to compare independent, or, at least, nearly independent, estimates from the same neuron. Due to the LRD, it is impossible to obtain such nearly independent estimates from biological neurons (i.e., it would require recording from the same neuron over time intervals that were infinitely separated). Thus, we used a model which could be restarted in such a way as to obtain independent estimates from the same "neuron" (i.e., keeping all of the parameters the same).

For our purposes, the model must be a point process in order to represent the times-of-occurrence of action potentials, and it must include the LRD observed in auditory-nerve activity. We used the fractional-Gaussian-noise-driven Poisson process (fGnDP) to model the long-range-dependent actionpotential trains found in AN fibers. This process was originally developed to model LRD action-potential trains in the AN (Teich 1989; Teich et al. 1990a, b; Teich 1992; Lowen and Teich 1993, 1995, 1996b, 1997; Kumar and Johnson 1993; Teich and Lowen 1994; Thurner et al. 1997; some of whom referred to this process as the "fractal-Gaussian-noise-driven Poisson process") and is arguably the simplest and most general model of LRD action-potential trains.

The fGnDP consists of a doubly stochastic Poisson process in which the initial stochastic process (i.e., the process determining the rate of the Poisson process) is fractional Gaussian noise (fGn). In other words, the fGnDP is an inhomogeneous Poisson process with samples of fGn specifying the time-varying rate. $\mathrm{fGn}$ is a generalization of the common white Gaussian noise that can be LRD (Mandelbrot 1965; Mandelbrot and van Ness 1968; Mandelbrot and Wallis 1968, 1969a, b, c; Beran 1994; Samorodnitsky and Taqqu 1994). It is a stochastic process (a sequence 
of random variables) in which the random variables have a particular correlation structure. This correlation structure is parameterized by the Hurst index $H$, which can vary from 0.0 to 1.0 . When $H<0.5$, fGn is statistically unstable and rarely useful for modeling natural processes. If $H=0.5$, then fGn is just white Gaussian noise. Hence, in this case, it has no temporal correlation and is not LRD. When $H>0.5$, fGn is LRD and the value of $H$ determines the strength of this LRD. As $H$ increases from 0.5 to 1.0 , the strength of the LRD increases. Thus, when $H>0.5$, the fGn imparts LRD to the fGnDP.

Standard fGn, which we denote by $G_{\mathrm{H}}(t)$, has a mean of 0 and a standard deviation of 1 . In our fGnDP models, the initial stochastic process, $\Lambda(t)$, determining the rate of the Poisson process was approximately a translated and scaled version of standard fGn, i.e.

$$
\Lambda(t) \approx \tilde{\Lambda}(t)=\lambda+\sigma \cdot G_{\mathrm{H}}(t),
$$

where $\lambda$ determines the mean of $\tilde{\Lambda}(t)$ and $\sigma$ determines the variance (or "noisiness") of $\tilde{\Lambda}(t)$. One problem arises, however, if the $\tilde{\Lambda}(t)$ is used as the actual rate process. This process can have negative values, and the rate of a Poisson process cannot be negative. The typical method for handling this situation is to rectify the translated and scaled fGn, so that the actual rate process $\Lambda(t)$ is formed by replacing all of the negative values that occur in $\tilde{\Lambda}(t)$ with zero. The resulting point process is what is commonly referred to as an fGnDP in the literature; we will use this same nomenclature. Because of the rectification procedure, $\lambda$ and $\sigma$ do not exactly specify the mean and variance, respectively, of $\Lambda(t)$ nor of the output discharge rate. The differences between the parameters and their corresponding statistics will increase with the number of negative values in the sample of $\tilde{\Lambda}(t)$.

We also used another model that is very similar to the fGnDP model described above, but which does not require rectification of the fGn driving noise. In order to make clear the relationship between the two models that we used, we will first briefly describe the method that we used to simulate the (inhomogeneous) Poisson process portion of the fGnDP. It is well known that a homogeneous Poisson process of unit rate can be converted to an inhomogeneous Poisson process with rate $\lambda(t) \geq 0$ by performing a time transformation based on $\lambda(t)$ (e.g., Cox and Lewis 1966; Cinlar 1975; Snyder 1975; Cox and Isham 1980; Taylor and Karlin 1994; Daley and Vere-Jones 2003; see the Appendix for mathematical details). Therefore, we first simulated a unit-rate homogeneous Poisson process using the fact that the interpoint intervals of this process are independent, identically distributed exponential random variables with a mean of one. We were then able to simulate the inhomogeneous Poisson process as a series of interpoint intervals using the following iterative procedure. Suppose that $t_{i}$ is either the starting time of the simulation or the time of occurrence of the last point produced by the simulation and that $e_{i}$ is a value drawn from a unit-mean exponential distribution. Then the time of occurrence of the next point in the simulation of an inhomogeneous Poisson process is the minimum time $t_{i+1}>t_{i}$ for which $\int_{t_{i}}^{t_{i+1}} \lambda(u) d u \geq e_{i}$. Thus, the interpoint interval of length $e_{i}$ in the homogeneous Poisson process is transformed to the interpoint interval of length $\left(t_{i+1}-t_{i}\right)$ in the inhomogeneous process. As long as the $e_{i}$ 's are all independent of one another, this procedure can be repeated indefinitely to produce an inhomogeneous Poisson process of rate $\lambda(t)$.

Now this procedure is reminiscent of a model that is very common in neuroscience: the integrate-andfire (IF) neuron. In the IF neuron, after a discharge occurs, the subsequent input is integrated until the value of the integral reaches a predetermined threshold, at which time the next discharge occurs and the process repeats itself. Hence, if the input to an IF neuron is the nonnegative function $\lambda(t)$, the threshold of the IF neuron changes after every discharge, and these thresholds are independently generated from an exponential distribution with unity mean, then the output of the IF neuron is an inhomogeneous Poisson process of rate $\lambda(t)$. We call this type of IF neuron a Poisson-equivalent IF model. Therefore, any inhomogeneous or doubly stochastic Poisson process may be conceptualized as the gray pathway in the block diagram of Figure 2A. (Of course, the rectification block is unnecessary if the rate function or stochastic rate process is nonnegative.) So, for instance, if the upper block in Figure 2A is the process $\tilde{\Lambda}(t)$ given in Eq. 1, a scaled and translated version of $\mathrm{fGn}$, then the gray pathway would be an fGnDP.

The Poisson-equivalent IF model can meaningfully operate on negative inputs, unlike the classical inhomogeneous Poisson process and other popular methods for simulating it. Thus, if we remove the rectification step, as in the black pathway in the block diagram of Figure 2A, we obtain a process that is identical to an inhomogeneous or doubly stochastic Poisson process for nonnegative inputs, but for which negative input values have a negative effect on the output. The negative input values reduce the value of the running integral that is accumulating toward the threshold $e_{i}$, and thus they extend the interval to the next spike $t_{i+1}$. Figures 2B and C illustrate the effect of negative input values on the Poisson-equivalent IF model. Suppose the upper block in Figure 2A generates the waveform shown in the upper graph 


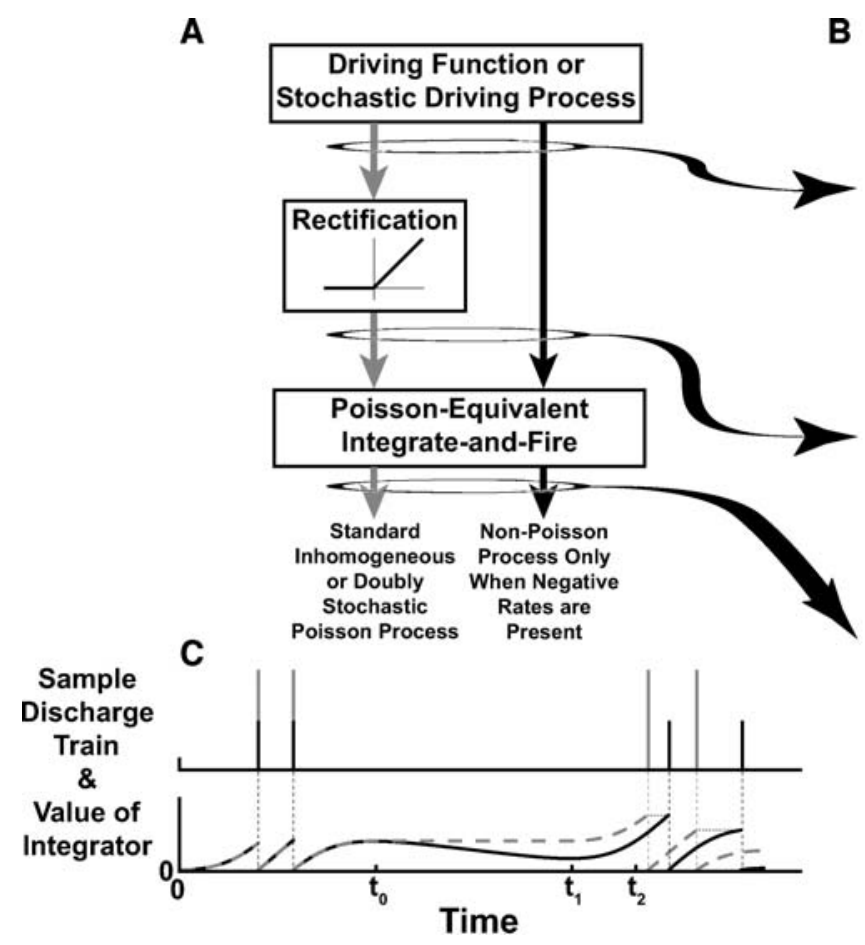

FIG. 2. Illustration of the difference between the two models used to simulate auditory-nerve discharge trains. A. Block diagram depicting a standard inhomogeneous or doubly stochastic Poisson process (gray pathway), in which the input must be rectified, and a Poisson-like process (black pathway), which can operate meaningfully on negative input values. If the output of the driving process (top block) does not contain negative values, then the two processes produce the same result, and both are inhomogeneous or doubly stochastic Poisson processes. B. Graphs describing the effect on the Poisson process (gray, dashed curves) and Poisson-like process

of Figure 2B. Then the curves in the middle graph are the inputs to the Poisson-equivalent IF block in Figure 2A for the rectifying model (gray pathway of Fig. 2A, gray, dashed curves in Fig. 2B) and the nonrectifying model (black pathway of Fig. 2A, black, solid curves in Fig. 2B), and the curves in the lower graph are the discharge rates that it would produce. These curves are identical prior to time $t_{0}$ because the driving function is nonnegative for that entire time, and they are both equal to zero from time $t_{0}$ to $t_{1}$ because the driving function is negative during that period. However, during the latter time period, the value of the integration mechanism decreases for the nonrectifying model, while it remains constant for the rectifying model (see lower graph of Fig. 2C). Thus, after time $t_{1}$, the output rate of the rectifying model is again identical to the driving function, which is nonnegative during this time. However, the integration mechanism of the nonrectifying model must first "recover" from its reduction during the previous time period. Because the two hatched regions shown in the upper graph of Figure 2B have the same area, the value of the integration mechanism

(black, solid curves) of the identical driving functions shown in the upper graph. The two hatched regions in the upper graph have equal area. C. A sample discharge train and the corresponding integrator-value curve from each model (gray "spikes" and dashed, gray curve for the Poisson process and black "spikes" and black, solid curve for the Poisson-like process) using the driving functions from $\mathbf{B}$ and identical sequences of randomly chosen threshold values in the Poisson-equivalent integrate-and-fire block. The "spikes" are differently sized to aid in visualization. See text for further description.

at time $t_{2}$ will be equal to its value at $t_{0}$. Thus, the output rate of the nonrectifying model is zero from time $t_{1}$ to $t_{2}$, a period during which the value of the integrator is less than its value at time $t_{0}$ (which was below threshold), but identical to the driving function (and the output rate of the rectifying model) after time $t_{2}$.

Figure 2C illustrates the difference between the rectifying (gray "spikes" and gray, dashed curve) and nonrectifying (black "spikes" and black, solid curve) models for a single sample discharge train resulting from the driving function of Figure 2B. The upper graph depicts the discharge train for each model as a set of "spikes," and the lower graph shows the value of the integrator for each model. For both models, the same sequence of threshold values was used in the Poisson-equivalent IF mechanism. Thus, because there are no negative values in the driving function before time $t_{0}$, both models discharge at the exact same times during this period. However, as a result of the decrease in the value of the integrator for the nonrectifying model between times $t_{0}$ and $t_{1}$, after time $t_{1}$ it will discharge later than the rectifying model. 
As mentioned above, if the upper block in Figure 2A is an fGn generator that outputs the process $\tilde{\Lambda}(t)$ given in Eq. 1, then the gray pathway, or rectifying model, would be an fGnDP. In this case, the black pathway, or nonrectifying model, would be an fGnDP-like process in which negative values in $\tilde{\Lambda}(t)$ have a negative effect on the output. We call this model the fractional-Gaussian-noise-driven Poissonequivalent-integrate-and-fire (fGnDP-IF) model. As is apparent from Figure 2A, the only real difference between the fGnDP and fGnDP-IF models is the presence or absence, respectively, of the rectification operation. Furthermore, although the IF mechanism in the fGnDP-IF seems to suggest a biological mechanism, this figure and the preceding discussion linking these two models demonstrate that this is not necessarily the case. Instead, the Poisson-equivalent IF mechanism is simply a mathematical construct for generating a Poisson process, which we were able to adapt for a more general class of inputs and which is not inherently any more suggestive than the Poisson process in the fGnDP.

Throughout this study, we compare the results from our models to the SR histogram of Liberman (1978) (Fig. 1), which is similar to other such histograms (e.g., Kiang et al. 1965; Joris and Yin 1992). Liberman's SR histogram was composed of estimates of the average SR from 738 individual AN fibers. In each fiber, the SR estimate was computed from the number of action potentials in a 30-s window. Liberman divided these measurements into three classes, low, medium, and high SR, corresponding approximately to the mass in the zero-to-one bin, the remainder of the lower mode, and the upper mode of the SR histogram, respectively. (Liberman actually used a boundary value of 0.5 spikes/s between the low-SR class and the medium-SR class, but, because his bins are 1 spike/s wide, this boundary is not apparent in the histogram.) In his study, $16 \%$ of the estimates were in the low-SR category, $23 \%$ were in the medium-SR category, and $61 \%$ were in the high-SR category.

Each of our model histograms is composed of the average discharge rates in 738 independent simulations, which matches the number of fibers in Liberman's SR histogram. The duration of each simulation was $30 \mathrm{~s}$. When three different SR categories (low, medium, and high) were simulated for a histogram, the percentages of these simulations in each category matched those found by Liberman. When only two SR categories (low and high) were simulated, the percentage in the high category was unchanged, and the percentage in the low category was $39 \%$, the total percentage (low SR + medium SR $=16 \%+23 \%$ ) in the lower mode of the SR histogram.

All of the simulations in a single category for a single histogram had identical parameter values. The parameters of the model are $\lambda, \sigma$, and $H . \lambda$ is approximately the mean discharge rate of the model and was the only parameter that varied between different SR categories within the same histogram. $\sigma$ is related to the variance of the (instantaneous) discharge rate and was usually set to be 25.1 spikes/s. In a previous study (unpublished), we found that the formula $\sigma=\sqrt{\lambda \cdot 9}$ worked well for matching the LRD statistics for simulations to those for AN fibers when $\lambda$ was large. When $\lambda=70$, the value that we used for the high-SR category, this formula yields a value for $\sigma$ of 25.1. $H$ is the Hurst index, which specifies the strength of the LRD in the model. For LRD simulations, $H$ was set to 0.9 . The Hurst indices estimated from AN fibers are typically in the range from 0.75 to 0.95 (Teich 1989, 1992; Teich et al. 1990a, b; Kelly et al. 1996; Lowen and Teich 1996a; note that some of these studies estimated the fractal dimension $D$ instead of the Hurst index $H$; however, these two measures are related by the expression $D=2 H-1)$. Estimates of $H$ are known to be negatively biased (e.g., Lowen and Teich 1993), so we chose a value in the upper portion of the range reported for $H$. As a control, we also ran non-LRD simulations with $H=0.5$. In this case, the variance of the driving process (i.e., the fGn) at a single point in time was identical to that in the LRD simulations, but there were no temporal correlations. This is a better control model than the Poisson process because the fGn, whether LRD or not, adds some variability to the discharge rate of the model.

\section{RESULTS}

The most common stochastic point-process model of neural action-potential trains, including those in the $\mathrm{AN}$, is the Poisson process. The Poisson process is to stochastic point processes what white Gaussian noise is to the more common "time-series" stochastic processes, having a simple statistical structure with no temporal correlations. Figure 3 contains a histogram of discharge-rate estimates calculated from 738 independent, 30-s-long simulations of Poisson processes. Approximately the same number of simulations had each of eight different rates: $0,5,10,20,40$, 60,80 , and 100 spikes/s. The width of the bins in this and the remainder of the histograms in this study is 1 spike/s to match that in Liberman's SR histogram (Fig. 1). Each mode of the histogram in Figure 3 is very narrow compared to the closest (in terms of rate) mode in the SR histogram of Figure 1. Hence, in order for this histogram to resemble the SR histogram, the distribution of the discharge rates of the Poisson processes would have to be fairly similar to the SR histogram. Therefore, if AN action-potential trains 


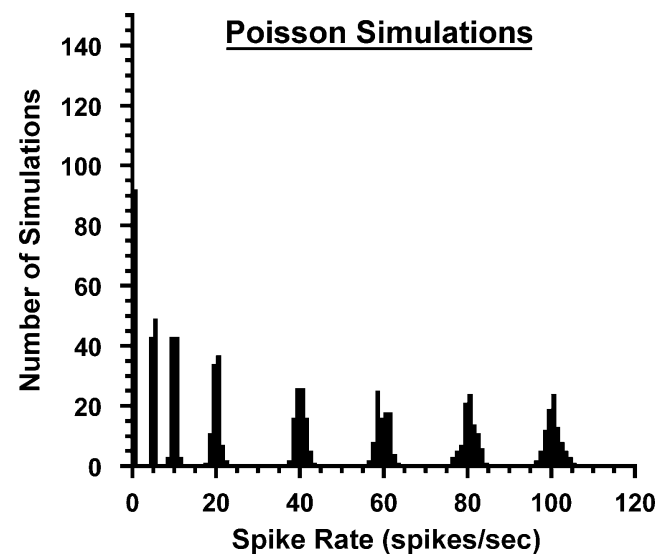

FIG. 3. Histogram of discharge rate estimates calculated from 738 independent, 30-s-long simulations of Poisson processes. Eight different rates, 0, 5, 10, 20, 40,60, 80, and 100 spikes/s, were used for the Poisson processes, and either 92 or $93(\sim 738 / 8)$ simulations were run at each of these rates. The width of the bins is $1 \mathrm{spike} / \mathrm{s}$.

were approximately Poisson, the SR histograms would correspond fairly well to the real distribution of SRs. Refractoriness would only narrow the modes of the histogram in Figure 3 and not significantly change this argument.

AN action-potential trains, however, are different than Poisson processes (with or without refractoriness) in that they are LRD. While temporal averaging can quickly reduce the variability of mean-rate estimates for short-range-dependent point processes (including processes with no temporal dependence, such as Poisson processes), the rate of increase in estimation reliability due to temporal averaging is exceptionally slow for LRD processes. Figure 4 illustrates this effect using simulations of a Poisson process and two versions of the fGnDP. One of the fGnDPs has a Hurst index of $H=0.5$ and does not, therefore, contain any temporal correlations, while the other has a Hurst index of $H=0.9$ and is, therefore, LRD. Both, however, have the same "instantaneous" variance, because the individual values of the fGn driving processes have the same variance in both cases. Thus, any statistical differences between these two fGnDPs are solely the result of LRD. Although the widths of the distributions of mean-rate estimates for the Poisson process and the non-LRD fGnDP are significant for a 1-s counting interval, with standard deviations of 8.4 and 11.4 spikes/s, respectively, they are considerably narrower for a 30 -s counting interval, with standard deviations of 1.5 and 2.1 spikes/s, respectively. And once the counting interval is 3,600 s (one hour) in length, the estimates only extend into the two ( $1 \mathrm{spike/s}$ ) bins on either side of the actual
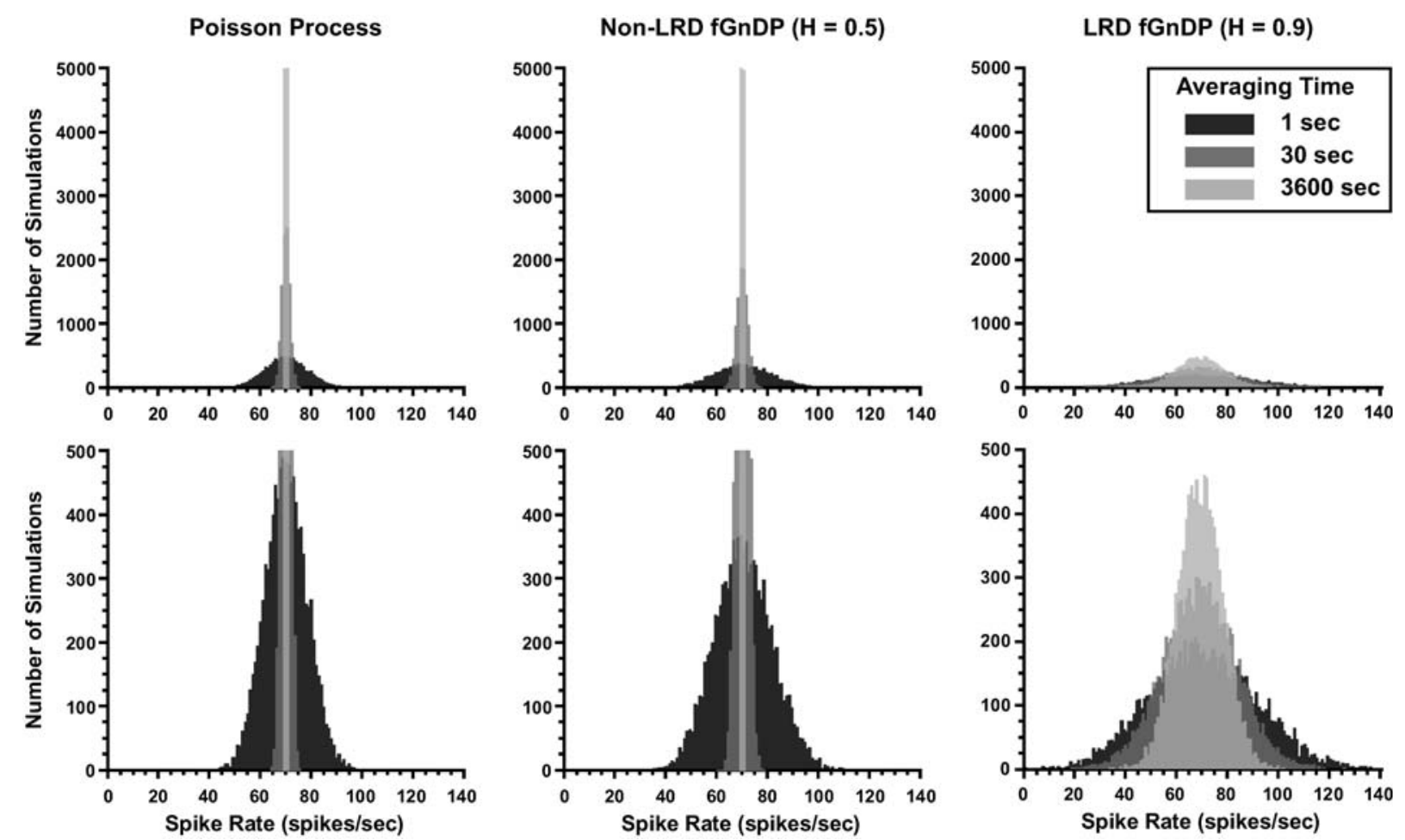

FIG. 4. Histograms of discharge rate estimates from 1-, 30-, and 3,600-s-long simulations of a Poisson process, a non-LRD fGnDP with Hurst index $H=0.5$ (i.e., no temporal correlations), and an LRD fGnDP with $H=0.9$. For each model, the upper and lower graphs are identical except for their vertical scales. Each histogram is the result of 10,000 independent simulations, each with a mean spike rate of $\lambda=70$ spikes/s. All fGnDP simulations had an fGn standard deviation of $\sigma=25.1$ spikes/s. The width of the bins is 1 spike/s. 
mean rate, with standard deviations of 0.14 and 0.19 spikes/s for the Poisson process and non-LRD fGnDP, respectively. Thus, the instantaneous variability of the fGn driving process in the fGnDP only moderately increases the variability of the mean-rate estimates and does not substantially alter the benefit gained by elongating the counting interval. However, the LRD in the other fGnDP model dramatically reduces the benefit of longer counting intervals, resulting in standard deviations of 21.5, 14.2, and 8.8 spikes/s for the 1-, 30-, and 3,600-s counting intervals, respectively. Thus, for this LRD point process, the variability of mean-rate estimates remains considerable even when the averaging time is one hour long!

Figure 4 illustrates that the variability of the meanrate estimates from a single-mean-rate model of auditory-nerve action-potential trains that incorporates LRD is comparable to the variability in the modes of SR histograms from auditory-nerve recordings, such as that shown in Figure 1. We were, therefore, interested in ascertaining how well a histogram of mean-rate estimates from simulations of this LRD model, using a minimal number of actual mean rates, would resemble auditory-nerve SR histograms, using Liberman's (1978) histogram (Fig. 1) as an archetype of these histograms. Because the SR histogram in Figure 1 has two modes, we first tried to model it using two types of model neurons, low-SR and high-SR, where all low-SR model neurons have the same mean discharge rate with $\lambda=1 \mathrm{spike/s}$ and all high-SR model neurons have the same mean discharge rate with $\lambda=70$ spikes/s. Figure 5 contains histograms for fGnDP model neurons with two mean discharge rates. The general shape of the histogram for the LRD model neurons is very similar to the SR histogram in Figure 1, except that the mass in the zero-to-one bin is missing. The histogram for the non-LRD model neurons, however, is quite different. In fact, the width of the modes in the non-LRD histogram is not significantly larger than that for the Poisson processes in Figure 3. This shows that the broadening of the modes in the right-hand (LRD) model histogram of Figure 5 is caused by the LRD and not by the extra "instantaneous" variance from the driving noise.

The most obvious method for producing the mass in the zero-to-one bin, which is missing in Figure 5, is to use three, instead of only two, types of model neurons. Figure 6 contains histograms for the fGnDP model with three types of model neurons: low-SR, medium-SR, and high-SR. The high-SR type is identical to the high-SR type in Figure 5, and the medium-SR type is identical to the low-SR type in Figure 5. However, the histograms in Figure 6 contain a third type, the low-SR model neurons that have $\lambda=-30$ spikes/s. Having a negative value for $\lambda$ may seem odd at first because the action-potential train cannot have a negative rate. Nevertheless, upon further consideration, it should seem reasonable. Such a model simply represents a spiking system where the mean of the (noisy) process driving the action-potential generator is well below the threshold of the generator, so that only strong positive deviations from the mean of the driving process actually cause action potentials to be generated. In this case, the mean of the driving process is nonlinearly related to, and only partially determinative of, the mean at the output, because of the threshold of the actionpotential generator. A similar effect on the histogram

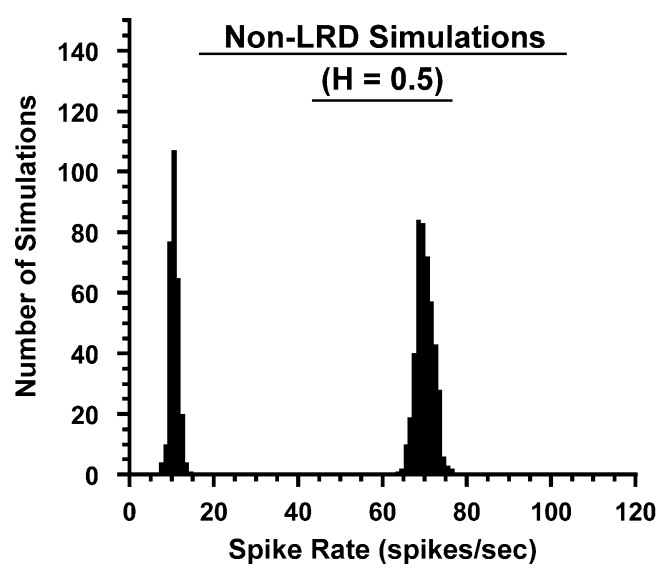

FIG. 5. Histograms of discharge rate estimates calculated from 30s-long simulations of fGnDPs (i.e., with rectified fGns) using two different mean rates. Each histogram is the result of 738 independent simulations: $288(\sim 39 \%)$ simulations with an fGn mean of $\lambda=1$ spike/s (low SR) and $450(\sim 61 \%)$ simulations with $\lambda=70$ spikes $/ \mathrm{s}$

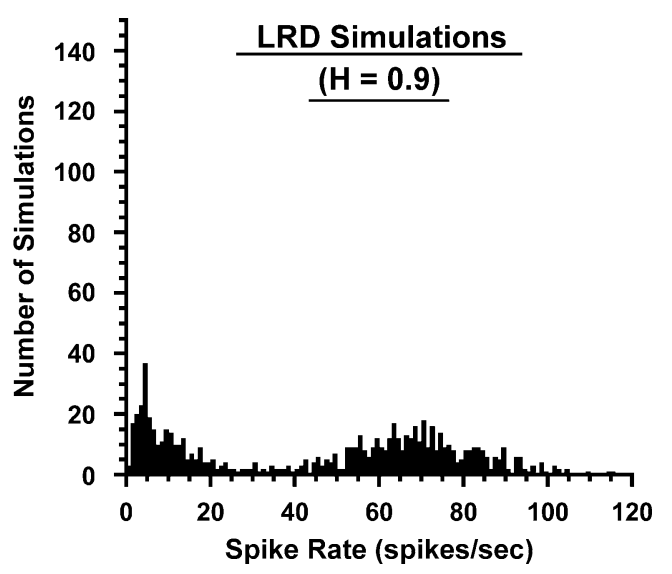

(high SR). The left histogram is the result from simulations of fGnDPs with Hurst index $H=0.5$ (not LRD), and the right histogram is the result from simulations of fGnDPs with $H=0.9$ (LRD). All simulations had an fGn standard deviation of $\sigma=25.1$ spikes/s. The width of the bins is 1 spike/s. 


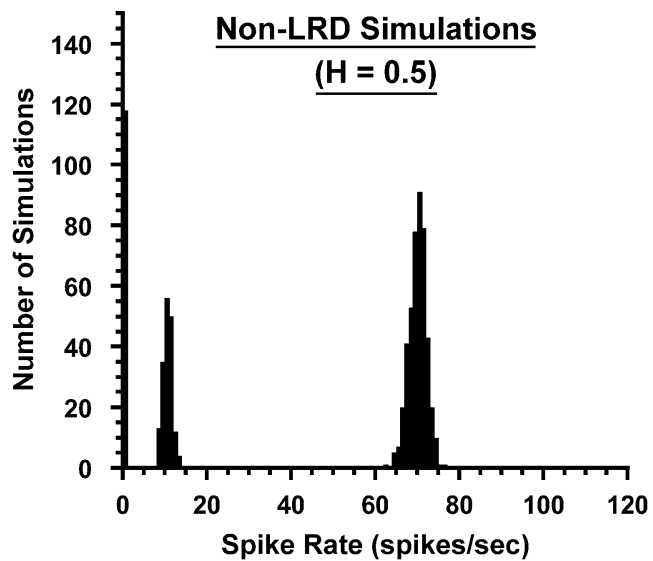

FIG. 6. Histograms of discharge rate estimates calculated from 30s-long simulations of fGnDPs (i.e., with rectified fGns) using three different mean rates. Each histogram is the result of 738 independent simulations: $118(\sim 16 \%)$ simulations with an fGn mean of $\lambda=-30$ spike/s (low SR), $170(\sim 23 \%)$ simulations with $\lambda=1$ spike/s (medium SR), and $450(\sim 61 \%)$ simulations with $\lambda=70$ spikes $/ \mathrm{s}$

can be produced by setting $\lambda$ between zero and one, and setting the parameter $\sigma$ to a very low value (e.g., 1 instead of 25.1) so that the discharge rate estimates at the output remain mostly within the zero-to-one bin. Except for the improvement of adding the "zero mass," the histograms in Figure 6 show essentially the same results as in Figure 5.

The striking resemblance between the LRD histogram in Figure 6 and Liberman's SR histogram in Figure 1 is particularly satisfying in that the parameters of the model were not fine-tuned in order to produce this result. In particular, the parameters affecting the width of the modes in the LRD histogram were chosen entirely a priori based on physiological considerations (see Methods).

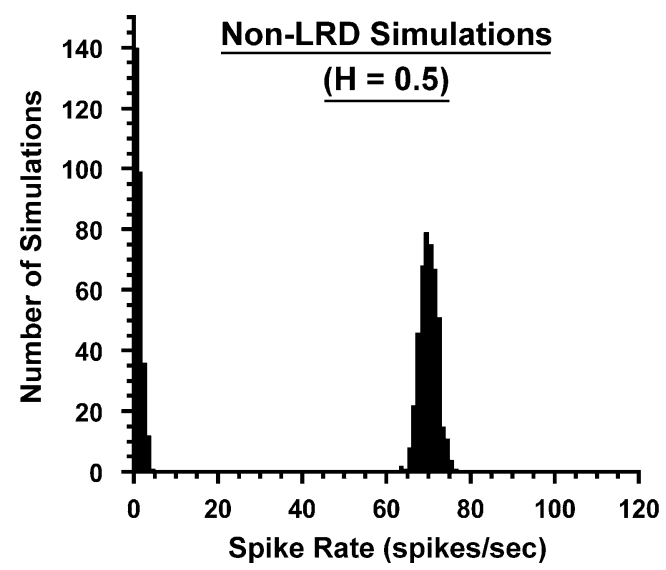

FIG. 7. Histograms of discharge rate estimates calculated from 30s-long simulations of fGnDP-IFs (i.e., with unrectified fGns) using two different mean rates. Each histogram is the result of 738 independent simulations: $288(\sim 39 \%)$ simulations with an fGn mean of $\lambda=1$ spike/s (low SR) and $450(\sim 61 \%)$ simulations with $\lambda=$

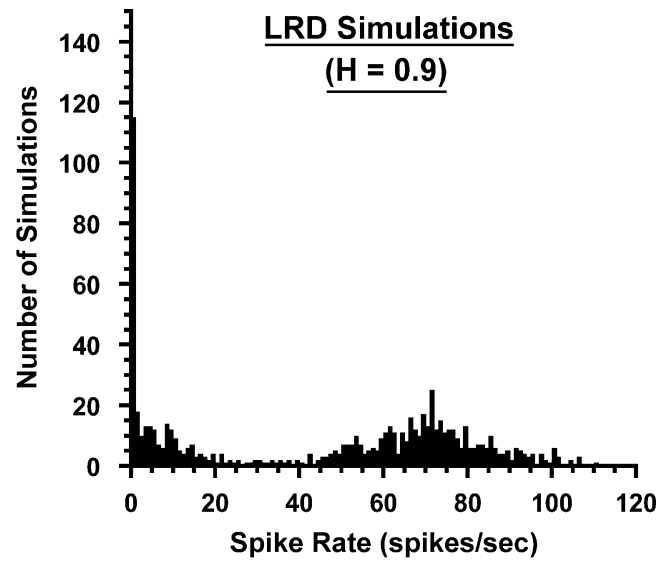

(high SR). The left histogram is the result from simulations of fGnDPs with Hurst index $H=0.5$ (not LRD), and the right histogram is the result from simulations of fGnDPs with $H=0.9$ (LRD). All simulations had an fGn standard deviation of $\sigma=25.1$ spikes/s. The width of the bins is 1 spike/s.

The reason that the "zero mass" does not appear in the LRD model histogram in Figure 5 is that negative values in the fGn driving noise do not have a negative effect on the discharge rate. Because they are simply set equal to zero by the rectification, their influence on the overall mean discharge rate-drawing it toward zero-is substantially weakened. In order to obtain more measurements in the "zero" bin of the histogram produced with only two types of model neurons, negative values of the fGn driving noise must have a negative effect on the rate of the Poisson process instead of being ignored. The negative effect of negative inputs is produced in the Poisson-equivalent IF process described in the Methods section, which is equiva-

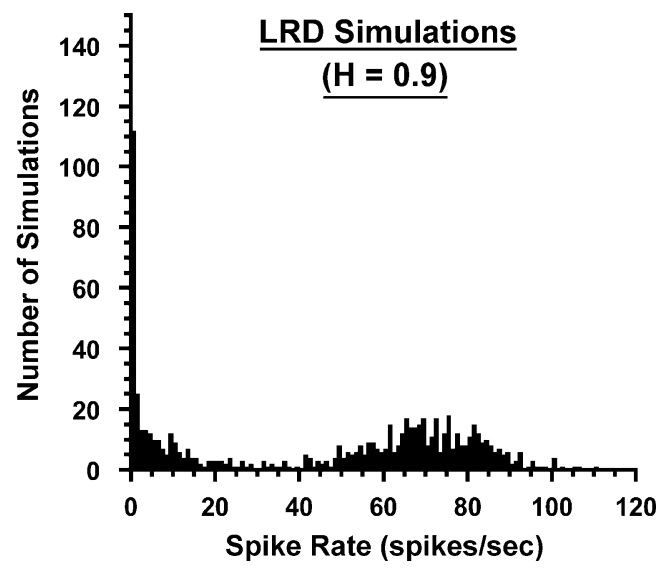

70 spikes/s (high SR). The left histogram is the result from simulations of fGnDP-IFs with Hurst index $H=0.5$ (not LRD), and the right histogram is the result from simulations of fGnDP-IFs with $H=0.9$ (LRD). All simulations had an $\mathrm{fGn}$ standard deviation of $\sigma=$ 25.1 spikes/s. The width of the bins is 1 spike/s. 
lent to the (inhomogeneous or doubly stochastic) Poisson process for nonnegative inputs but also operates on negative inputs.

Figure 7 contains the histograms for the fractionalGaussian-noise-driven Poisson-equivalent-integrateand-fire (fGnDP-IF) model with only two types of model neurons, where all low-SR model neurons have the same mean discharge rate with $\lambda=1$ spike $/ \mathrm{s}$ and all high-SR model neurons have the same mean rate with $\lambda=70$ spikes/s. These simulations were the same as those in Figure 5, except that the fGn driving noise was not rectified (i.e., the black pathway in Fig. 2A was used for these simulations, while the gray pathway was used for those in Fig. 5). As expected, this model created a mass in the zero-to-one bin, even when only two different discharge rates were used in the simulations. As in Figure 6, the histogram for the LRD model neurons is remarkably similar to Liberman's SR histogram (Fig. 1), while the histogram for the non-LRD model neurons is not at all similar to the SR histogram. Again, the parameters affecting the width of the modes in the LRD histogram were chosen entirely a priori based on physiological considerations.

\section{DISCUSSION}

Typical SR histograms for AN fibers, such as that shown in Figure 1, seem to suggest that there are two or three classes of AN fibers and that, at least in two of these classes, the spontaneous rates are widely distributed. This would indeed be the case if AN action-potential trains were statistically similar to Poisson processes, but, on the contrary, AN actionpotential trains are known to be long-range dependent (LRD). Our simulations of simple LRD models for AN action-potential trains show that the broad modes in SR histograms can be produced by the variability in average discharge rate estimates caused by LRD and are not necessarily indicative of broad distributions of SRs themselves. In fact, presupposing the presence of LRD comparable to that found by other researchers, our results prove that SR histograms similar to that in Figure 1 would be produced even if all of the neurons within a single SR class have identical mean SRs. Moreover, our results demonstrate not only the possibility that there is a minimal number of different mean SRs in the AN, but also the likelihood that this is the case. Otherwise, the SR histograms from the AN would be much broader than they actually are, because the variability of SR estimates is necessarily a combination of the variability of the actual SRs and the additional variability in the estimates, most of which in this case is due to the LRD. Therefore, we conclude that the broadness of the modes in experimentally derived SR histograms from AN fibers is an artifact of measurement variability produced by LRD and is not indicative of a broad distribution of actual mean spontaneous discharge rates.

Unfortunately, there is no way to circumvent the effects of LRD in order to better ascertain the "true" spontaneous rate of an AN fiber from a finite-length sample of its action-potential train. As Figure 4 suggests, an extremely long averaging time would be required in order to obtain a reliable estimate of the "true" spontaneous rate from an LRD action-potential train. Moreover, using the formula for the variance of counts of an fGnDP that was derived in Jackson (2003), we are able to compute confidence intervals for the sample mean for unusually long averaging times, and the results are not very encouraging. For an fGnDP with an approximate mean of $\lambda=70$ spikes $/ \mathrm{s}$, an approximate standard deviation of $\sigma=25.1$ spikes $/ \mathrm{s}$, and a Hurst index of $H=0.9$, the $95 \%$ confidence intervals of the sample mean rate for averaging times of an hour, a day, a week, and a month are, in spikes/s, $(49.4,90.6),(55.0,85.0)$, $(57.7,82.3)$, and $(61.2,78.8)$, respectively. For a similar non-LRD fGnDP with $H=0.5$, the widths of these confidence intervals are all less than 1 spike/s wide, with the width of the smallest being less than $0.01 \mathrm{spikes} / \mathrm{s}$.

Spontaneous rate is apparently correlated with many other properties of AN fibers, such as threshold, input-output function shape, morphology, spatial location, and projection sites (e.g., Kiang et al. 1965, 1970, 1976; Sachs and Abbas 1974; Liberman 1978, 1980, 1982, 1991, 1993; Rhode and Smith 1985; Ryugo and Rouiller 1988; Kawase and Liberman 1992; Ryugo and May 1993; Leake et al. 1993). Thus, our results suggest that many of these properties may be fairly uniform within each SR class. Measurements of electrophysiological properties, such as threshold and input-output function shape, however, typically have significant variability within a single SR class. Undoubtedly, some of this variability is attributable to differences in cochlear mechanics between different locations on the basilar membrane and between different individuals. Nevertheless, there is likely to be additional variability in these measurements associated with LRD. The estimates of any property measured using absolute action-potential counts, such as saturation rate or the rate of response to any particular stimulus, are probably going to be affected by LRD in the same way that estimates of spontaneous rate are, greatly decreasing the meaningfulness of the precise values calculated.

On the other hand, measurements based on the difference between the counts in nearby time intervals, such as many typical methods for measuring 
threshold or the shape of input-output functions, should not be directly affected by LRD in the process. Yet, there may be causal connections between these properties and the action-potential counts that would suggest that these properties are inherently LRD. The estimates of these properties would thus necessarily be affected by LRD. For instance, the thresholds of AN fibers may vary over time and be LRD, and this may be the cause of the LRD in AN spontaneous activity. In this situation, much of the variability in the threshold estimates from a single SR class of AN fibers would be attributable to the LRD and would not indicate inherent differences between these AN fibers.

The study of anatomical properties may be a more promising way to further support, or challenge, our results. These properties would need to be ones that are fairly stable within single AN neurons. Otherwise, they may vary over time in relation to the spontaneous rate, and, thus, measurements of such properties at instants in time would be highly variable just like estimates of spontaneous rate. A number of studies (e.g., Liberman 1980, 1982, 1991, 1993; Ryugo and Rouiller 1988; Kawase and Liberman 1992; Ryugo and May 1993; Leake et al. 1993) have found anatomical and morphological characteristics that differ between AN fibers in different SR classes and, therefore, are evidence for different SR classes. For the most part, however, the results from these studies do not help to clarify whether or not SR is widely distributed within each class. The one exception is Figure 2 in Liberman (1982), which contains plots of both AN fiber diameter and position versus SR. Although this figure clearly supports the conclusion that these two properties of AN fibers differ between the SR classes, there do not appear to be any trends with SR within each individual SR class. This lends some, albeit weak, support that SR is narrowly distributed within each SR class. Additional findings of discrete anatomical classes of AN fibers with homogeneity of stable anatomical properties within these classes would further strengthen our argument for the limited variability of spontaneous rate within each class. However, strong anatomical support of, or opposition to, this argument would require experimental investigation of the distribution of anatomical properties that are directly related to the "true" spontaneous rate of AN fibers, something that would be very difficult to determine.

Our models and analysis are incapable of determining whether there are two or three different spontaneous rates in the AN. The primary issue is what causes the large mass in the zero-to-one-spikesper-second bin of AN SR histograms (see Fig. 1). We found that this mass could be created by either adding a third SR class or by altering the Poissonprocess model slightly so that it could operate meaningfully on negative-valued inputs. The latter seems to be the more parsimonious explanation, especially because the Poisson process is a mathematical construct that is only associated to neural actionpotential trains through the statistics of the output and not the mechanism itself. The mechanism of the Poisson-equivalent IF process that we have introduced is a more satisfying explanation of AN actionpotential generation because it relies on the integrate-and-fire neuron model, a common model of action-potential generation in many systems. Furthermore, it contains the minimal rectification necessary for an action-potential generation model, that which is inherent to the production of discharges itself, and does not require rectification of the driving process. The economy and physiologically realistic mechanism of this model support the hypothesis of only two SR classes. Another argument for the two-class hypothesis is that the data from which Liberman (1978, see Figs. 10 and 12) originally concluded that there is a separate SR class below 0.5 spikes/s can be well explained in another way. This data exhibited a sharp increase in the variability of threshold measurements in AN fibers with SR measurements that were nearly zero spikes/s. Assume that threshold measurements are (negatively) correlated with short-term SR measurements, whether or not they are good estimates of the "true" SR. Yet, as the threshold increases beyond the point that is associated with zero spikes/s, the SR measurements will no longer decrease due to rectification, whether implicit in the action-potential generation or explicit elsewhere in the system. Thus, all of the would-be "negative SRs" become near-zero SRs, which are therefore associated with a wide range of thresholds. In this case, the AN fibers with nearzero SR measurements (Liberman's low-SR units) do not form a class distinct from the AN fibers with higher SR measurements up to about 18 spikes/s (Liberman's medium-SR units), but the large variability in threshold measurements from AN fibers with near-zero SR measurements is produced.

Our Poisson-equivalent IF model may also be a useful alternative to the inhomogeneous Poisson process in other studies of spiking systems where the driving function is capable of assuming negative values. This model introduces more opportunity for extension and refinement as they become necessary. It can be readily extended, when necessary, by drawing on the long history of neural models that have been formed through modification of the basic IF mechanism. Furthermore, the more intuitive structure of this model lends itself well to refinement. For instance, the LRD could be incorporated into the firing mechanism itself by making the thresholds LRD, and this may ultimately provide a better representation of the actual physiological mechanism. 


\section{ACKNOWLEDGMENT}

This research was supported by NIH grant R01 DC01641.

\section{APPENDIX}

As mentioned in the Methods section, a homogeneous Poisson process of unit rate can be converted to an inhomogeneous Poisson process of rate $\lambda(t)>0$ by means of a time transformation. More specifically, if $M(t)$ is a unit-rate homogeneous Poisson process, $\lambda(t)$ is nonnegative, and the function $\tau$ is defined as $\tau(t)=\int_{0}^{t} \lambda(u) \mathrm{d} u$, then $N(t) \equiv M(\tau(t))$ is an inhomogeneous Poisson process of rate $\lambda(t)$ (e.g., Cox and Lewis 1966; Cinlar 1975; Snyder 1975; Cox and Isham 1980; Taylor and Karlin 1994; Daley and Vere-Jones 2003). Thus, a unit-rate homogeneous Poisson process is converted to an inhomogeneous Poisson process of rate $\lambda(t)$ by making the time transformation $t \mapsto \tau^{-1}(t)$, where at each time $t$ the value of $\tau^{-1}(t)$, the inverse function of $\tau$, is the smallest value of $s$ for which $\tau(s) \geq t$.

\section{REFERENCES}

Beran J. Statistics for Long-Memory Processes. Chapman \& Hall/ CRC Press, Boca Raton, FL, 1994.

Cinlar E. Introduction to Stochastic Processes. Prentice-Hall, Englewood Cliffs, NJ, pp. 95-97, 1975.

Cox DR, Isнam V. Point Processes. Chapman \& Hall, London, p. 48, 1980.

Cox DR, Lewis PAW. The Statistical Analysis of Series of Events. Methuen, London, pp. 28-29, 1966.

Daley DJ, Vere-Jones D. An Introduction to the Theory of Point Processes. Springer-Verlag, New York, pp. 22-23, 2003.

JACKSON BS. Consequences of long-range temporal dependence in neural spike trains for theories of coding and processing. PhD thesis, Syracuse University, Syracuse, NY, 2003.

JACKSON BS. Including long-range dependence in integrate-and-fire models of the high interspike-interval variability of cortical neurons. Neural Comput. 16(10):2125-2195, 2004.

Joris PX, YIN TC. Responses to amplitude-modulated tones in the auditory nerve of the cat. J. Acoust. Soc. Am. 91:215-232, 1992.

KaWASE T, Liberman MC. Spatial organization of the auditory nerve according to spontaneous discharge rate. J. Comp. Neurol. 319:312-318, 1992.

Kelly OE, Johnson DH, Delgutte B, Cariani P. Fractal noise strength in auditory-nerve fiber recordings. J. Acoust. Soc. Am. 99:2210-2220, 1996.

Kiang NyS, Watanabe T, Thomas C, Clark LF. Discharge Patterns of Single Fibers in the Cat's Auditory Nerve. MIT Press, Cambridge, MA, 1965.

Kiang NYS, Moxon EC, Levine RA. Auditory-nerve activity in cats with normal and abnormal cochleas. In: Wolstenholme GEW, Knight J (eds) Sensorineural Hearing Loss. Churchill, London, pp. 241-273, 1970.

Kiang NYS, Liberman MC, Levine RA. Auditory-nerve activity in cats exposed to ototoxic drugs and high-intensity sounds. Ann. Otol. Rhinol. Laryngol. 85:752-768, 1976.
Kumar AR, Johnson DH. Analyzing and modeling fractal intensity point processes. J. Acoust. Soc. Am. 93:3365-3373, 1993.

LEAKE PA, SNyder RL, HRADEK GT. Spatial organization of inner hair cell synapses and cochlear spiral ganglion neurons. J. Comp. Neurol. 333:257-270, 1993.

Liberman MC. Auditory-nerve response from cats raised in a lownoise chamber. J. Acoust. Soc. Am. 63(2):442-455, 1978.

Liberman MC. Morphological differences among radial afferent fibers in the cat cochlea: An electron-microscopic study of serial sections. Hear. Res. 3(1):45-63, 1980.

Liberman MC. Single-neuron labeling in the cat auditory nerve. Science 216:1239-1241, 1982

Liberman MC. Central projections of auditory-nerve fibers of differing spontaneous rate. I. Anteroventral cochlear nucleus. J. Comp. Neurol. 313:240-258, 1991.

Liberman MC. Central projections of auditory nerve fibers of differing spontaneous rate. II. Posteroventral and dorsal cochlear nuclei. J. Comp. Neurol. 327:17-36, 1993.

Lowen SB, Teich MC. Estimating the dimension of a fractal point process. Proc. SPIE 2036:64-76, 1993.

Lowen SB, Teich MC. Estimation and simulation of fractal stochastic point processes. Fractals 3:183-210, 1995.

LOWEN SB, TEICH MC. The periodogram and Allan variance reveal fractal exponents greater than unity in auditory-nerve spike trains. J. Acoust. Soc. Am. 99(6):3585-3591, 1996a.

Lowen SB, Teich MC. Refractoriness-modified fractal stochastic point processes for modeling sensory-system spike trains. In: Bower JM (eds) Computational Neuroscience. Academic Press, San Diego, pp. 447-452, 1996b.

Lowen SB, Teich MC. Estimating scaling exponents in auditorynerve spike trains using fractal models incorporating refractoriness. In: Lewis ER, Long GR, Lyon RF, Narins PM, Steele CR, Hecht-Pointar E (eds) Diversity in Auditory Mechanics. World Scientific, Singapore, pp. 197-204, 1997.

Mandelbrot BB. Une classe de processus stochastiques homothetiques a soi: application a loi climatologique de H. E. Hurst. C. R. Acad. Sci. Paris 240:3274-3277, 1965.

Mandelbrot BB, van Ness JW. Fractional Brownian motions, fractional noises and applications. SIAM Rev. 10:422-437, 1968.

Mandelbrot BB, Wallis JR. Noah, Joseph and operational hydrology. Water Resour. Res. 4:909-918, 1968.

Mandelbrot BB, Wallis JR. Computer experiments with fractional Gaussian noises. Water Resour. Res. 5:228-267, 1969a.

Mandelbrot BB, Wallis JR. Some long-run properties of geophysical records. Water Resour. Res. 5:321-340, 1969b.

Mandelbrot BB, WaLlis JR. Robustness of the rescaled range $R / S$ in the measurement of noncyclic long run statistical dependence. Water Resour. Res. 5:967-988, 1969c.

Rhode WS, Sмiтн PH. Characteristics of tone-pip response patterns in relationship to spontaneous rate in cat auditory nerve fibers. Hear. Res. 18:159-168, 1985.

Ryugo DK, MAY SK. The projections of intracellularly labeled auditory nerve fibers to the dorsal cochlear nucleus of cats. J. Comp. Neurol. 329:20-35, 1993.

Ryugo DK, Rouiller EM. Central projections of intracellularly labeled auditory nerve fibers in cats: Morphometric correlations with physiological properties. J. Comp. Neurol. 271:130-142, 1988.

Sachs MB, Abbas PJ. Rate versus level functions for auditory-nerve fibers in cats: Tone-burst stimuli. J. Acoust. Soc. Am. 56:1835-1847, 1974.

SAmorodnitsky G, TAQQU MS. Stable Non-Gaussian Random Processes: Stochastic Models with Infinite Variance. Chapman \& Hall/CRC, Boca Raton, 1994.

SNYDER DL. Random Point Processes. Wiley, New York, pp. 62, 115, 1975. 
Taylor HM, Karlin S. An Introduction to Stochastic Modeling. Academic Press, San Diego, pp. 246-247, 1994.

TEICH MC. Fractal character of the auditory neural spike train. IEEE Trans. Biomed. Eng. 36(1):150-160, 1989.

Tегсн MC. Fractal neuronal firing patterns. In: McKenna T, Davis J, Zornetzer SF (eds) Single Neuron Computation. Academic Press, Boston, pp. 589-625, 1992.

Teich MC, Lowen SB. Fractal patterns in auditory nerve-spike trains. IEEE Eng. Med. Biol. Mag. 13(2):197-202, 1994.

Teich MC, Johnson DH, Kumar AR, Turcott RG. Rate fluctuations and fractional power-law noise recorded from cells in the lower auditory pathway of the cat. Hear. Res. 46:41-52, 1990a.

Teich MC, Turcott RG, Lowen SB. The fractal doubly stochastic Poisson point process as a model for the cochlear neural spike train. In: Dallos P, Geisler CD, Matthews JW, Ruggero MA, Steele CR (eds) The Mechanics and Biophysics of Hearing. Springer-Verlag, New York, pp. 354-361, 1990b.

Thurner S, Lowen SB, Feurstein MC, Heneghan C, Feichtinger HG, Teich MC. Analysis, synthesis, and estimation of fractal-rate stochastic point processes. Fractals 5:565-596, 1997. 